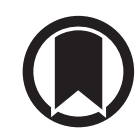

CrossMark

\title{
Impact of chest imaging quality on the diagnosis of the usual interstitial pneumonia pattern: a hub and spoke study
}

To the Editor:

Accurate diagnosis of idiopathic pulmonary fibrosis (IPF) is essential to ensure prompt initiation of appropriate treatment and enrolment in clinical trials. According to current international guidelines [1], in the appropriate clinical context the identification of the usual interstitial pneumonia (UIP) pattern on chest high-resolution computed tomography (CT) allows a confident diagnosis of IPF. Patients referred to tertiary centres for suspected interstitial lung disease (ILD) may provide CT images that do not fulfil recommended scanning protocols [1], therefore triggering the execution of another CT. However, the impact of suboptimal technical quality on the identification of the UIP pattern by thoracic radiologists is unknown. We assessed the quality of chest CT scans performed in the context of an ILD network including a tertiary ILD centre (hub) and the affiliated centres (spokes), and the impact on the identification of the UIP pattern.

Patients were retrospectively identified from those referred to the ILD clinic of the University Hospital Gemelli in Rome (hub centre) between March 2014 and October 2017. Patients were enrolled in the study if they had two consecutive chest CT scans within a 12-month time interval, one performed at the hub centre and a previous one at a spoke centre.

Chest CT scans were randomised and assessed for technical quality by an expert thoracic radiologist using currently recommended scanning protocols [1], including the level of inspiration or expiration, the use of sharp or standard reconstruction filters, slice thickness and degree of motion artefacts. Examinations were scored using a five-level system for overall image quality, ranging from 1 (very poor) to 5 (very high). Two thoracic radiologists with different level of expertise in ILD (18 and 4 years, respectively) assessed CT scans and assigned each to one of the following five categories [1]: UIP, probable UIP, indeterminate for UIP, alternative diagnosis, non-fibrotic. The diagnostic categories were then converted to a binary "UIP versus any of the other categories" score. Individual CT features, namely honeycombing, reticulation, traction bronchiectasis and ground glass opacities, were also scored. Final diagnosis for each patient was based on a multidisciplinary discussion made at the hub centre after having performed the second CT scan [1].

Statistical analysis was performed using SPSS software package (version 24, IBM, USA). Data were summarised using means and standard deviations or counts and percentages, as appropriate. The Chi-squared test was used to assess statistical significance of proportions. Cohen's weighted $\mathrm{k}$ coefficient $\left(\mathrm{k}_{\mathrm{w}}\right)$ was used to express intra- and inter-observer agreement for diagnostic categories, while Cohen's unweighted $\mathrm{k}$ coefficient $(\mathrm{k})$ was used for the binary CT pattern scores. The level of agreement was categorised as follows: poor $\left(0<\mathrm{k}_{\mathrm{w}} \leqslant 0.20\right)$, fair $\left(0.20<\mathrm{k}_{\mathrm{w}} \leqslant 0.40\right)$, moderate $\left(0.40<\mathrm{k}_{\mathrm{w}} \leqslant 0.60\right)$, good $\left(0.60<\mathrm{k}_{\mathrm{w}} \leqslant 0.80\right)$ and excellent $\left(0.80<\mathrm{k}_{\mathrm{w}} \leqslant 1.00\right)$ [2].

76 patients were enrolled in the study. Most $(n=39,51.3 \%)$ patients received a final diagnosis of IPF. Seven (9.2\%) patients were diagnosed with chronic hypersensitivity pneumonitis and 13 (17.1\%) were deemed

@ERSpublications

CT technical quality affects the recognition of the UIP pattern by thoracic radiologists. Optimal CT scanning protocols should be implemented in spoke centres to speed up the diagnostic work-up of IPF, reducing costs and further radiation exposure. http://bit.ly/2Jo4EqQ

Cite this article as: Sgalla G, Larici AR, Re A, et al. Impact of chest imaging quality on the diagnosis of the usual interstitial pneumonia pattern: a hub and spoke study. Eur Respir J 2019; 53: 1900084 [https:// doi.org/10.1183/13993003.00084-2019]. 
unclassifiable. Other diagnoses were connective tissue-related ILD ( $n=4,5.3 \%)$, organising pneumonia $(n=4,5.3 \%)$, respiratory bronchiolitis-related ILD $(n=3,3.9 \%)$, idiopathic nonspecific interstitial pneumonia $(n=2,2.6 \%)$, sarcoidosis $(n=1,1.3 \%)$, alveolar proteinosis $(n=1,1.3 \%)$, occupational ILD $(n=1$, $1.3 \%)$ and pleuroparenchimal fibroelastosis $(n=1,1.3 \%)$. Mean age was $71 \pm 9.8$ years and there was a male predominance $(n=49,64.5 \%)$. Most $(n=49,65.3 \%)$ patients had history of smoking. On first clinical presentation at the hub centre, mean per cent predicted forced vital capacity (FVC) was $86.4 \pm 25.2 \%$ pred, while mean diffusing capacity of the lung for carbon monoxide (DLCO) was $60.2 \pm 23.3 \%$ pred. Mean time between consecutive CTs was $6.3 \pm 2.7$ months.

Among CT scans performed at the spoke centres, 41 (53.9\%) had an overall image quality scored from very poor to moderate, while quality was high to very high in $72(94.7 \%)$ of CT scans performed at the hub centre $(\mathrm{p}<0.001) .46(60.5 \%)$ spoke exams were performed with sharp filter and section thickness $<1.5 \mathrm{~mm} ; 14$ (18.5\%) had moderate to high motion artefacts and only seven (9.2\%) had expiratory scans.

The UIP pattern was identified by both observers more frequently in the hub than in the spoke CT scans (table 1). The UIP was the pattern most frequently diagnosed by the more experienced radiologist, who identified about $20 \%$ more UIP patterns at the hub centre, both in the overall study population and in patients with a final diagnosis of IPF (table 1). In contrast, the probable UIP was the pattern most frequently identified by the less experienced radiologist (table 1). The inter-observer agreement for the binary score of UIP pattern was similar for the hub and the spoke CT datasets $(\mathrm{k}=0.56$ and $\mathrm{k}=0.63$, respectively). The level of intra-observer agreement across the diagnostic categories in hub and spoke CTs was good for the more experienced radiologist $\left(\mathrm{k}_{\mathrm{w}}=0.70,95 \%\right.$ CI $\left.0.58-0.83\right)$, and moderate for the less experienced radiologist $\left(\mathrm{k}_{\mathrm{w}}=0.41,95 \%\right.$ CI $\left.0.24-0.58\right)$. The intra-observer agreement for the binary UIP score was good $(k=0.76)$ for the more experienced radiologist and fair $(k=0.35)$ for the less experienced one. There was good to optimal intra-observer agreement for the presence of honeycombing $(\mathrm{k}=0.83$ and $\mathrm{k}=0.67$ for more and less experienced radiologist, respectively) and reticulation ( $\mathrm{k}=0.77$ and $\mathrm{k}=0.70)$; there was lower agreement for the presence of traction bronchiectasis $(\mathrm{k}=0.47$ and $\mathrm{k}=0.60)$ and ground glass opacities $(\mathrm{k}=0.25$ and $\mathrm{k}=0.44)$.

We assessed the clinical impact of CT scanning protocols in the context of a hub and spoke ILD network comprising a specialised centre for ILD and its affiliated centres. The results show how lower CT image quality in spoke centres may affect the identification of the UIP pattern, even by an expert thoracic radiologist.

High-quality scanning protocols are strongly recommended to allow accurate classification of the CT patterns into one of the four diagnostic categories [1,3]. Thin sections $(<2 \mathrm{~mm})$, high spatial resolution kernel, full inspiration to total lung capacity, volumetric CT acquisition, and expiratory scans are all required parameters to ensure optimal image quality [3]. Patients referred to specialised centres for diagnosis and management of ILD often undergo a second chest CT scan, possibly because their initial chest CT, often performed at a non-specialised centre, is considered suboptimal. Although it is not clear whether this approach may enhance diagnostic accuracy, it exposes patients to additional ionising radiation, increases costs and delays diagnosis.

TABLE 1 Usual interstitial pneumonia (UIP) diagnostic patterns on computed tomography scans performed at spoke and hub centres in the overall study population $(n=76)$ and in patients with a diagnosis of idiopathic pulmonary fibrosis (IPF) ( $n=39$ )

\begin{tabular}{|c|c|c|c|c|c|c|}
\hline & \multicolumn{3}{|c|}{ More experienced radiologist } & \multicolumn{3}{|c|}{ Less experienced radiologist } \\
\hline & Spoke & Hub & p-value & Spoke & Hub & p-value \\
\hline All patients & & & $<0.001$ & & & $<0.001$ \\
\hline UIP & $22(28.9 \%)$ & $28(36.8 \%)$ & & $12(15.8 \%)$ & $14(18.4 \%)$ & \\
\hline Probable UIP & $22(28.9 \%)$ & $20(26.3 \%)$ & & $25(32.9 \%)$ & $22(28.9 \%)$ & \\
\hline Indeterminate for UIP & $9(12.9 \%)$ & $9(11.8 \%)$ & & $14(18.4 \%)$ & $15(19.7 \%)$ & \\
\hline Alternative diagnosis & $14(18.4 \%)$ & $12(15.8 \%)$ & & $12(15.8 \%)$ & $14(18.4 \%)$ & \\
\hline Non-fibrotic & $9(11.8 \%)$ & $7(9.2 \%)$ & & $13(17.1 \%)$ & $11(14.5 \%)$ & \\
\hline IPF patients & & & $<0.001$ & & & NS \\
\hline UIP & $18(46.2 \%)$ & $23(59.0 \%)$ & & $10(25.6 \%)$ & $12(30.8 \%)$ & \\
\hline Probable UIP & $15(38.5 \%)$ & $12(30.8 \%)$ & & $17(43.6 \%)$ & $14(35.9 \%)$ & \\
\hline Indeterminate for UIP & $3(7.7 \%)$ & $4(10.3 \%)$ & & $8(20.5 \%)$ & $6(15.4 \%)$ & \\
\hline Alternative diagnosis & $3(7.7 \%)$ & 0 & & $3(7.7 \%)$ & $6(15.4 \%)$ & \\
\hline Non-fibrotic & 0 & 0 & & $1(2.6 \%)$ & $1(2.6 \%)$ & \\
\hline
\end{tabular}


The base finding of our study was the fact that several technical requirements for optimal CT scan were not fulfilled by examinations performed at spoke centres. Notably, the overall image quality was significantly lower as compared to scans performed at the hub centre. The difference in the proportions of UIP pattern reported by the two observers, in both the overall study population and in IPF patients, indicates that a high level of experience in thoracic imaging is crucial for a more confident detection of UIP and diagnosis of IPF. Notably though, the higher proportions of UIP pattern found in hub CT scans suggest that the assessment of the radiological features essential for the diagnosis of UIP is facilitated by the implementation of adequate scanning protocols. Importantly, a similar difference in the proportions of UIP pattern between hub and spoke CTs was found in patients with IPF, suggesting that suboptimal CT quality may ultimately impact the multidisciplinary discussion diagnostic process. Radiologists with fewer years of experience may be particularly biased towards the identification of UIP and honeycombing on low quality CT scans, suggested by the lower levels of intra-observer agreement. In particular, the combination of a less experienced radiologist reviewing lower quality CT scans resulted in a 57\% relative decrease in the detection of UIP as compared to a more experienced radiologist reviewing optimal quality CT scans.

Our study has some limitations, such as the small size of the population, the limited number of thoracic radiologists involved and the fact that both radiologists worked at the hub centre. Moreover, it cannot be excluded that the CT pattern may have changed between the two consecutive CT scans, although the average interval between exams was short (6 months).

Nonetheless, these findings indicate that a more proactive effort by scientific societies is desirable to standardise CT scanning protocols across referral and non-referral centres, thus facilitating the prompt recognition of the UIP pattern, speeding up the diagnostic work-up, and reducing costs and radiation exposure. This cost-effective intervention might ultimately increase the overall efficiency of the diagnostic process for IPF.

Giacomo Sgalla $\oplus^{1}$, Anna Rita Larici $^{2,3}$, Antonina $\mathbf{R e}^{4}$, Alessandra Farchione ${ }^{3}$, Giuseppe Cicchetti ${ }^{2,3}$, Lucio Calandriello $^{3}$, Alessia Comes ${ }^{1}$, Nicoletta Golfi ${ }^{1}$, Bruno Iovene ${ }^{1}$, Francesco Varone ${ }^{1}$, Riccardo Manfredi ${ }^{2,3}$ and Luca Richeldi ${ }^{1,5}$

${ }^{1}$ Dipartimento di Scienze Cardiovascolari e Toraciche, Fondazione Policlinico Universitario A. Gemelli IRCCS, Rome, Italy. ${ }^{2}$ Istituto di Radiologia, Università Cattolica del Sacro Cuore, Rome, Italy. ${ }^{3}$ Dipartimento Diagnostica per Immagini, Radioterapia Oncologica ed Ematologia, Fondazione Policlinico Universitario A. Gemelli IRCCS, Rome, Italy.

${ }^{4}$ Azienda Ospedaliero-Universitaria Ospedali Riuniti di Ancona, Ancona, Italy. ${ }^{5}$ Istituto di Medicina Interna e Geriatria, Università Cattolica del Sacro Cuore, Rome, Italy.

Correspondence: Giacomo Sgalla, Unità Operativa Complessa di Pneumologia, Fondazione Policlinico Universitario A. Gemelli IRCCS, Università Cattolica del Sacro Cuore, Largo Agostino Gemelli 8, 00168 Roma, Italy. E-mail: giacomo. sgalla@gmail.com

Received: Jan 142019 | Accepted after revision: March 172019

Conflict of interest: G. Sgalla reports personal fees from Boehringer Ingelheim, outside the submitted work. A.R. Larici has nothing to disclose. A. Re has nothing to disclose. A. Farchione has nothing to disclose. G. Cicchetti has nothing to disclose. L. Calandriello has nothing to disclose. A. Comes has nothing to disclose. N. Golfi has nothing to disclose. B. Iovene has nothing to disclose. F. Varone has nothing to disclose. R. Manfredi has nothing to disclose. L. Richeldi reports personal fees for consultancy from Sanofi-Aventis, ImmuneWorks, Celgene, Nitto and Bristol Myers Squibb, personal fees for advisory board work from Roche, Fibrogen and Promedior, personal fees for lecturing from Shionogi, personal fees for steering committee work from Boehringer Ingelheim, personal fees for editorial activities from DynaMed, outside the submitted work.

Support statement: The study was funded in part by the Fondazione Policlinico A. Gemelli IRCCS, Rome, Italy. A. Re was supported by a research grant from the Italian Respiratory Society.

\section{References}

1 Raghu G, Remy-Jardin M, Myers JL, et al. Diagnosis of idiopathic pulmonary fibrosis: an official ATS/ERS/JRS/ ALAT clinical practice guideline. Am J Respir Crit Care Med 2018; 198: e44-e68.

2 Brennan P, Silman A. Statistical methods for assessing observer variability in clinical measures. BMJ 1992; 304: 1491-1494.

3 Lynch DA, Sverzellati N, Travis WD, et al. Diagnostic criteria for idiopathic pulmonary fibrosis: a Fleischner Society White Paper. Lancet Respir Med 2018; 6: 138-153. 